\title{
ZIELE
}

\section{In zehn Schritten zu einem Leitbild der 3. Generation}

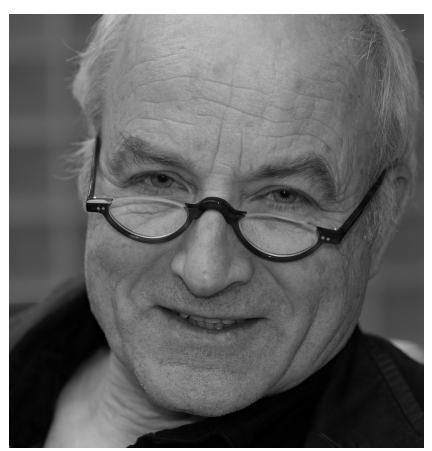

VON ANDREAS STRUNK Prof. Dr. phil. Andreas Strunk, Dipl. Ing. ist Sozialpädagoge und Sozialplaner. In der Gesellschaft für Innovation, Systementwicklung und Soziale Arbeit (GISAmbH) leitet er Projekte der Organisationsentwicklung und Sozialforschung. Weiterbildungen als Supervisor, lösungsorientierter Berater und Case Manager. Er ist Vorstandsmitglied im Deutschen Berufsverband für Soziale Arbeit (DBSH), Landesverband BadenWürttemberg. prof.strunk@t-online.de

\author{
Gesucht werden Leitbilder der 3. Generation; \\ diese orientieren sich am Modell der "Lernenden \\ Organisation«. Die Leitbilder der 3. Generation \\ versuchen, organisationales Lernen in Verbindung \\ zu bringen mit individuellem Lernen, um damit \\ die Anpassungsfähigkeit der Organisation \\ oder des Unternehmens an sich verändernde \\ Kontextbedingungen zu ermöglichen.
}

Den Trägern der Sozialwirtschaft wird häufig eine besondere Funktion im Sozialstaat zugewiesen. Man erwartet von ihnen eine besondere ethische Qualität bei der Gestaltung ihrer Angebote. Solche Zuschreibungen sind beispielsweise: "Wertegarantenfunktion « (Serries und Hübinger, 1991), „Werte Elite« (Storz) und »Megatrend Werte« (Suliková und Olma, 2012). Die beiden letztgenannten Autoren formulieren bezogen auf organisationale Konsequenzen der Werthaltungen eine Reihe von Anfragen, beispielsweise

- Sind Leitbilder in der Lage entsprechend handlungsleitend zu sein?

- Gibt es Konflikte zwischen Werteund Dienstleistungsorientierung?

- Wie wichtig ist die Wertorientierung des Trägers zur Profilierung und Abgrenzung?

- Wie sieht das Wertemanagement aus und in wessen Zuständigkeit liegt es?

Der Dreh- und Angelpunkt der Beantwortung dieser Fragen liegt aus meiner Sicht in den Modalitäten der Leitbildentwicklung und der Funktion von Leitbildern in der Organisationsentwicklung, die Leistungsanbieter in der Sozialwirtschaft versuchen. Entsprechende Erfahrungen liegen vor, wie sie in einer neuen Veröffentlichung des Nomos-Verlages zum Thema »Leitbildentwicklung und systemisches Controlling « versammelt sind (Strunk u.a. 2012).

Leitbilder der 2. Generation werden als Performanz-Leitbilder bezeichnet (Hess 2007), so ein Ergebnis einer Studie des Fraunhofer-Institutes für Arbeitswirtschaft und Organisation. Diese Leitbilder sind im »Gegenstrom-Verfahren « als Vermittlung zwischen oberer Leitungsebene und Mitarbeitenden der Basis erarbeitet worden. Die Konzentration auf die Ebene des konkreten Arbeitshandelns ist das wesentliche Kennzeichen der Performanz-Leitbilder.

Leitbilder der 3. Generation orientieren sich am Modell der "Lernenden Organisation « (Senge 2011), indem sie versuchen, organisationales Lernen in Verbindung zu bringen mit individuellem Lernen. Damit soll die Anpassungsfähigkeit der Organisation oder des Unternehmens an sich verändernde Kontextbedingungen ermöglicht werden. Leitbilder der 3. Generationen definieren Ziele und sichern deren Überprüfbarkeit. Die Leitbilder werden durch ebenenübergreifende Diskussionsprozesse überprüft und weiterentwickelt.

Die Vorstellung der Generationenabfolge sollte nicht zu eng auf "alt« und "neu « fokussieren: Vorstellungen $\mathrm{zu}$ Leitbildern der 3. Generation sind schon vor zehn Jahren entwickelt und erprobt worden (Pering 2003).

Anhand des fiktiven Unternehmens 
»Stadtdiakonie Curo (SDC) « soll vorgestellt werden, wie ein Verfahren zur Leitbildentwicklung der 3. Generation abgelaufen kann. Zum Zeitpunkt des Beginns der Leitbildentwicklung beschäftigte die Organisation rund 400 Mitarbeiterinnen und Mitarbeiter. Der Jahresumsatz lag bei 14 Millionen Euro. Der Auftrag zur Leitbildentwicklung kam vom Vorstand. Eine Lenkungsgruppe wurde eingerichtet. Sie wurde so besetzt, dass sich ein einigermaßen repräsentativer Querschnitt über alle Abteilungen und vorhandenen Einrichtungen ergab.

1. Schritt: Befragung mittels Inter- viewleitfäden. Für den Vorstand war es wichtig, dass der Erfahrungsstand der Mitarbeitenden bezogen auf die SDC-Praxis deutlich werden konnte, und zwar im Sinne einer Ressourcenund Lösungsorientierung: Was können wir besonders gut? Was macht uns gegenüber den uns anvertrauten Menschen besonders attraktiv? Was müssen wir lernen?

2. Schritt: Auswertung durch die 2. Beratungsfirma. Die Interviews wurden durchgesehen und in Großgruppenveranstaltungen mit den Mitarbeitenden und den Leitungskräften diskutiert. Hier ergaben sich erste Impulse sowohl für organisationales als auch individuelles Lernen.

3. Schritte: Verdichtung der Er3. gebnisse im Zielkatalog. Sechs Zielbereiche wurden identifiziert: anvertraute Menschen, Mitarbeitende, Gemeinden des Kirchenbezirkes, politische Gemeinde, Kostenträger, andere Träger. Für jeden wurden Ziele benannt, deren Verfolgung für das Gelingen der SDC-Praxis wichtig erschien.

4. Schritt: Bewertung des Zielkataloges. In der Leitbild-Lenkungsgruppe wurden die Ziele gewichtet und Vorschläge zur Operationalisierung gemacht, die für die SDC-interne Organisationsentwicklung relevant wurden.

5 Schritt: Orientierungsrahmen. 5. Beim »Orientierungsrahmen " handelt es sich um den Versuch einer Zusammenfassung dessen, was bisher erarbeitet wurde: also Bestandsaufnahme, organisationale Treiber (»Was können wir besonders gut? «), Ziele und Merkmale der Zielerreichung. Leitbild und Vision waren bisher noch nicht unmittelbar thematisiert. Im Orientierungsrahmen wurden erste Entwürfe für Leitbild und Vision deutlich und diskutiert. Es gab drei Fassungen und entsprechende Diskussionsverläufe zwischen der Leitbildlenkungsgruppe und dem Vorstand. Die Orientierungsrahmen wurden durch die Beratungsfirma erstellt.

6. Schritt: Erörterung im Verwaltungsrat. Der Verwaltungsrat als oberste Ebene stimmte schließlich dem Leitbild-Entwurf in einer Kurz- und einer Langfassung zu. Kleinere Veränderungen wurden erbeten und berücksichtigt.

7. Schritt: Erörterung in den Einrichtungen. Für die Erörterung in den Einrichtungen wurden verschiedene Wege gewählt. Einerseits gab es wieder Großgruppenveranstaltungen, die thematischen Schwerpunkte für die Erörterung des Leitbildes ermöglichten und andererseits wurden die Einrichtungen gebeten, das SDC-Leitbild zum Anlass zu nehmen, eigene (einrichtungsspezifische) Leitbilder zu erarbeiten.

8. Schritt: Vision und Leitbild. 8. Die Vision wurde im Prinzip als Zielerreichung im Sinne der Ziele aus den Zielbereichen verstanden. Ein Beispiel aus dem Zielbereich »Gemeinden des Kirchenbezirkes«: »Die Beachtung dieser Ziele mobilisiert ehrenamtliche Kräfte und finanzielle Ressourcen als verbindende und absichernde Elemente zwischen kirchlicher Praxis und beruflicher Diakonie.« Auf diese Weise sind Aussagen des Leitbildes mit Aussagen der Vision verbunden.

9. Schritt: Überprüfung der Brauchbarkeit von Vision und Leitbild. Elemente des Leitbildes werden zum Anlass genommen, Maßnahmen der Organisationsentwicklung zu ergreifen. So gibt es beispielsweise Angebote zum »Führungskräftetraining «: Unterstützung bei der Formulierung eigener Führungsprinzipien, Ausarbeitung von Führungsdatensätzen, Gesprächsführung bei Konflikten etc. Auf diese Weise gibt es eine Überprüfbarkeit »on the job«.
10. Schritt: Konsequenzen für Controlling und Organisationsentwicklung. Neben den genannten Impulsen für die Organisationsentwicklung gibt es zur Zeit Anlässe zur Überprüfung des Qualitätsmanagements (»Schlachtet die heiligen Kühe!«) und zur Qualifizierung des Beratungsverhaltens bei den Fachkräften des SDC-Controllings. Das hängt mit dem doppelten Mandat des Controlling zusammen: einerseits Kontrollfunktion, andererseits Servicefunktion zur Verbesserung der Strukturen.

Das beschriebene Verfahren passt auf die spezifische Ausgangslage der genannten Organisation. Andere Unternehmen benötigen vermutlich andere Vorgehensweisen. Es gibt kein lückenlos generalisierbares Verfahren der Leitbildentwicklung. Es gibt lokale Theorien und lokale Richtigkeiten, die beachtet werden müssen, wenn eine Organisationsentwicklung auf die Balance von »Strukturverantwortung « und "Selbstorganisation« setzen will (Baitsch 1993).

\section{Resümee}

Leitbilder der 3. Generation sind handlungsleitend, weil sie überwiegend aus der Handlungspraxis der Mitarbeitenden abgeleitet werden. Es gibt keine Konflikte zwischen Werte- und Dienstleistungsorientierung, weil die Gestaltung der Dienstleistung (Uno-Acta-Beziehung) im Mittelpunkt der Aufmerksamkeit liegt. Da die Wertorientierung in engem Zusammenhang zur Dienstleistungsorientierung steht, ist sie zur Profilierung der Organisation und damit zur Abgrenzung zu konkurrierenden Trägern von entscheidender Bedeutung.

In der Konzeption unserer Beispielsorganisation kann es nicht nur eine $\mathrm{Zu}$ ständigkeit für das Wertemanagement geben. Die doppelte Engführung ( $\mathrm{Zu}-$ ständigkeit und Methode) in der Fragestellung ist problematisch. Gelingendes Wertemanagement ist das Ergebnis gelingender Organisationspraxis und insofern das Ergebnis einer erfolgreich lernenden Organisation.

Die genauen Literaturhinweise können der erwähnten Neuerscheinung entnommen werden: Andreas Strunk (Hg.): Leitbildentwicklung und systemisches Controlling. Nomos Verlagsgesellschaft, Baden-Baden 2012. 200 Seiten. 29,Euro. ISBN 978-3-8329-6473-3. 